\title{
Comparison of the Effect of Dexmedetomidine and Propofol on Duration of Starting Tremor after Drug discontinuation in Parkinson's Patients under Deep Brain stimulation
}

\author{
Faranak Behnaz ${ }^{1}$, Mahshid Ghasemi ${ }^{2} \&$ Yalda Shabanpour ${ }^{3}$ \\ ${ }^{1}$ Assistant Professor of Anesthesiology Shohada Tajrish Hospital, Shahid Beheshti University of Medical Science, \\ Iran \\ ${ }^{2}$ Assistant Professor of Anesthesiology Taleghani Hospital, Shahid Beheshti University of Medical Science, Iran \\ ${ }^{3}$ Resident of Anesthesiology Shohada Tajrish Hospital, Shahid Beheshti University of Medical Science, Iran \\ Correspondence: Mahshid Ghasemi, Assistant professor of Anesthesiology Taleghani Hospital, Shahid Beheshti \\ University of Medical Science, Tehran, Iran. E-mail: mahshidghasemi@sbmu.ac.ir
}

Received: December 6, 2017

Accepted: December 16, 2017

Online Published: January 1, 2018

doi:10.5539/jmbr.v8n1p1

URL: https://doi.org/10.5539/jmbr.v8n1p1

\begin{abstract}
The purpose of this study was to compare the effects of Dexmedetomidine and Propofol on the duration of starting tremor after drug discontinuation in patients with Parkinson's disease under deep brain stimulation. In order to reach the research goals, 28 individuals with Parkinson's disease under deep brain stimulation were randomly assigned into two groups including Dexmedetomidine and Propofol that referring to Assistant Professor of Anesthesiology Taleghani Hospital in 2016. In the implementation phase. The following drugs were used: Dexmedetomidine (Precedex) with dose of 0.2-0.4 $\mu / \mathrm{kg} / \mathrm{h}$ and Propofol with doses of 20-30 mg bolus, and then $10-20 \mu / \mathrm{kg} / \mathrm{min}$ infusion, with a control and preservation of BIS between 65 and 85, and also, RAMSY Sedation Score equal to 3. Locus of pin and the head scalp were anaesthetized with lidocaine drug. The total dose of local anaesthetic drugs was recorded at the end of the procedure. The interval time between drug discontinuation and start of tremor, and also, the time of emergence, was recorded as the time between relaxation and when the patient is able to cooperate for a neurological examination. All of the events during the operation and complications and complains of pain and restlessness were recorded. Moreover, the following cases were investigated Post-operative pain, vital signs, and hemodynamic changes, respiratory and alert status of patients for 2 hours in PACU. Patients' pain was measured using VAS (0: painless, 10: maximum pain) at intervals of 30 minutes. Independent t-test and Mann-Whitney test were used for data analysis. Findings showed that there was a significant difference between time to tremor and time to cooperation variables in the two groups. Mean comparison showed that the mean time to tremor and time to cooperation in the Propofol group was higher than the Dexmedetomidine (DEX) group. Other findings showed that there was no significant difference between BIS in the two groups. The results also showed that there was a significant difference between VAS and surgeon satisfaction variables in the two groups. Mean VAS in Propofol group was significantly higher than DEX group. Mean of surgeon satisfaction in the DEX group was significantly higher than Propofol group. In general, it can be said that patients with Parkinson's disease, Dexmedetomidine is superior drug to Propofol to deep brain stimulation.
\end{abstract}

Keywords: Parkinson's disease, deep brain stimulation, dysfunction, satisfaction, treatment

\section{Introduction}

Parkinson's is a degenerative disease of the basal ganglia, which is characterized by a defect in the secretion of dopamine and thus, reduced inhibition of the extrapyramidal motor system. Patient voluntary movements is decreased and usually have rigidity, resting tremors, masked faces, difficulty walking and talking, depression and dementia (Fábregas et al., 2002). Also, autonomic system dysfunction may occur with orthostatic hypotension, increased salivation and impairment of body temperature regulation. Because of difficulty in swallowing and changes in consciousness, these patients are at the risk of respiratory complications, aspiration, and muscular dysfunction, as well. Standard treatments include levodopa (often combined with carbidopa), anticholinergic drugs, bromocriptine, amantadine, and Selegiline. Levodopa can cause dyskinesia (involuntary movements of myoclonic and dystonic). Parkinson's etiology has not yet been identified. Parkinson's is seen in 3\% of people over 66 years 
of age. The classic tremor triad in resting situation, muscular rigidity and bradykinesia are due to dopaminergic in the dense part. Patients may refer to procedures for the treatment of Parkinson's disease or for the treatment of other surgical diseases (Maltête et al., 2004).

Parkinson's patients have a high risk of post-operative thrombosis that leads to accumulation of secretion and risk of aspiration. It may have autonomic instability, which can be due to Parkinson's disease, the condition of the treatment, or both. Exacerbation of the disease can lead to complications of muscular dysfunction, including respiratory distress and increased tremor, Akinesia, confusion or hallucinations. In general, anti-Parkinson drugs should be continued until surgery (Fábregas et al., 2002). The common drug, levodopa, has a short half-life, and its therapeutic effects may be reduced to the end of surgery. Other common causes in Parkinson's treatment include monoamine oxidase inhibitors, which interact negatively with opiate. Opiates may exacerbate muscular rigidity. Use of some drugs such as Phenothiazines, Butyrophenones, and Metoclopramide should be avoided because it may exacerbate Parkinson's symptoms. Propofol may have unpredictable effects on motor function and should be avoided in stereotactic procedures (Böhmdorfer, Schwarzinger, Binder, \& Sporn, 2003).

Deep Brain Stimulation is a surgical technique used to treat multiple neurodegenerative symptoms such as motor symptoms of Parkinson's disease, such as tremors, stiffness, difficulty in reducing movement, and walking problems. This method is also used to treat primary and secondary tremor. Currently, this method is used only for people who cannot adequately control the symptoms with drug (Maltête et al., 2004). Before surgery, MRI and CT scan are used to the precise identification of target location in the brain. The goals that are considered in procedures related to motor disorders are mainly: Sub-thalamic Nucleus, internal segment of the Globus Palidus, Ventral Intermediate Nucleus of the Thalamus (Finelli et al., 2002). The mechanism of its effect is not well defined. One theory is abnormal motor patterns that are caused by synchronous oscillations in neural circuits that involve the cortex and several basal ganglia nuclei, is created and stimulating multiple points along a path with a high frequency can interfere with the oscillations. Cases to be considered during anesthesia include: limitation of airway by Frame, limitation of the use of sedative medicines during electrophysiologic monitoring, diagnosis and treatment of complications (especially seizure and intrahepatic hematoma) (Schulz, Keh, Barner, Kaisers, \& Boemke, 2007). There are different approaches to this type of surgery. In some centers, it is preferable not to use any drug for anesthetizing patients and only with local anesthesia and complete patient wake up, this procedure is performed due to believe that anaesthetic drugs may interfere with the process of brain stimulation and prevent from obtaining good result.

In other cases, anaesthetic drugs are used especially Propofol and Dexmedetomidine, (Anderson, Marks, \& Futter, 1994; Guldenmund et al., 2017). To stimulate thalamic and sub- thalamic, part of the localization process requires the identification of a specific electrophysiological effect on a specific nucleus. Because the nature and duration of the effect of anaesthetic drugs are unclear, some surgeons and electrophysiologists ask that no drug be given. Patient tremor is a final point to decide on the adequacy of the stimulator and should not be suppressed by sedative agents; such suppression may be caused by Propofol (Anderson et al., 1994). Nonetheless, Rozet et al. successfully reported electrophysiologic records and preservation of the tremor when Dexmedetomidine was used to deploy a deep brain stimulator in patients with Parkinson's disease (Rozet, 2008; Rozet et al., 2006; Souter et al., 2007). Dexmedetomidine exerts its sedation and sleep effects with action on $\alpha 2$ receptors in the nucleus of locus coeruleus. The analgesic effect of Dexmedetomidine is exerted by the action on $\alpha 2$ receptors in the locus coeruleus and spinal cord (Yang, Xue, \& Sun, 2017).

Dexmedetomidine also decreases the projection activity of Locus coeruleus into the ventral preoptic nucleus and release of GABA and Galanin in the Tuberomammillary nucleus, and leads to a decrease in the release of histamine in cortical and Sub cortical projections. Alpha-2 agonists inhibit ion transfer through calcium channels of L-type and P-type, and facilitate ion transport through calcium-activated potassium channels (Mack, Perrine, Kobylarz, Schwartz, \& Amp; Lien, 2004; Schilling et al., 2017). Dexmedetomidine produces different types of receptors in comparison with other alternative drugs such as Propofol and benzodiazepines, which impose their effects through the GABA system, and also cause normal sleep patterns affects on the endogenous pathways of sleep. Patients who have been treated with Dexmedetomidine under the intubation of the chip can easily wake up and have the ability to co-operate properly and follow the instructions (Martinez-Simon et al., 2017; Souter et al., 2007). In a study (2004) on patients with Parkinson's disease and deep brain stimulation, deep corneal stimulations were performed in only using local anaesthetic with lidocaine in 15 patients who had a proper co-operation and were able to sustain intraoperative events.

In other 15 patients who had been matched for age and duration of the disease, due to severe anxiety and intolerance and proper co-operation, DBS was performed with general anaesthetic and intravenous Propofol injection (Maltête et al., 2004; Souter et al., 2007). In this study, it is clear that the severity of motor disability in both groups of 
patients has been clearly decreased, but the intensity of stimulation and the extent of residual motor disorder in the group under general anesthesia were greater, which indicates that stimulation of the subthalamic nucleus will have less accurate if clinical evaluation do not perform during surgery. This result is not shown in another study comparing the AWAKE stereotactic STN stimulation with General Anesthesia. In this study, there was no significant difference in the rate of post-operative improvement, or STN stimulation amplitude (Souter et al., 2007). In several studies, the effect of general anesthesia with Dexmedetomidine has been studied. Some of these studies suggest this drug as an ideal anaesthetic drug in patients with deep brain stimulation, as it is possible for patients to be awakened and clinical evaluation during surgery, while patients are ailing and relaxing (Schulz et al., 2007). Therefore, the purpose of the present study was to evaluate the effect of Dexmedetomidine and Propofol on the duration of starting tremor after drug discontinuation in Parkinson's patients under deep brain stimulation.

\section{Materials and Methods}

The type of study was randomized controlled study. The research population was all patients with Parkinson's disease under deep brain stimulation referring to Shohada Tajrish Hospital in year 2016. Through the available sampling method, 28 patients were divided in two groups: Dexmedetomidine and Propofol. These drugs are obtained from Operation room of Shohada Tajrish Hospital.

The criteria for entering the study included: age of 40-80 years old with Parkinson's disease, the lack of known drug sensitivity, ASA Class $1 \& 2$, Mallampati Score less than 3, normal head and neck examination, and lack of difficulty in ventilation and intubation of airway and Missing cases comprising Pregnancy, Breast-feeding women, allergy or absolute contraindication of any drug, chronic pain history, history of major chronic depression, BMI> 35 , concomitant illnesses such as diabetes, coronary heart disease, cerebrovascular accidents, hypertension, malignancy, failure Kidney, liver dysfunction, coagulation disorders and obstructive sleep apnea .... Ethical criteria of this study were approved by the ethics committee of Medical Sciences University of Shahid Beheshti.

\subsection{Manner of Execution}

Patients were fully informed about the procedure and the cases before entering the patients to study and they were informed in writing about their participation in the study. In this study, the patients were randomly divided into intervention and control groups. In the intervention group, Dexmedetomidine was used and in the other group intravenous Propofol was used. Using Excel and randomized numbers table, each patient was given a random number and each patient was assigned to one of the two groups according to their number. Basic information and clinical data of patients include general information about patients (age, sex, height, weight), clinical and paraclinical characteristics of patients, and also associated illnesses such as hypertension, diabetes, history of smoking and narcotics, Dementia, obstructive sleep apnea, gastroesophageal reflux, and mallampati score, musculoskeletal problems and ASA classification were recorded before the operation and in the questionnaire.

At the time of entering patients to operation room, the baseline heart rate (HR), non-invasive blood pressure (SPO2), respiratory rate (RR), BIS, and at intervals of 5 minutes, were recorded in the operating room. The rate of intraoperative bleeding in the patients was recorded in patients' files. All patients received oxygen ( 2 to $4 \mathrm{l} / \mathrm{min})$ through a nasal cannula. Suction, Nasal Airway, Oral Airway, LMA, and tracheal tube were available as essential equipment for possible airway conditions. Spo2<92\% and RR were less than or equal to 8 per minute. Suction, nasal airway, oral Airway, LMA, and tracheal tube were used as available essential equipment for possible airway conditions. The periods of spo2 $<92 \%$ and RR less than or equal to 8 per minute were recorded. Maneuvers and Actions required to manipulate and open the airway were recorded. The difference of $20 \%$ in primary blood pressure and heart rate whether decreased or whether increased, as well as the use of hypotensive drugs or inotrope drugs and vasopressor drugs were recorded, as well. The following drug were used: Dexmedetomidine (Precedex) with the dose of $0.2-0.4 \mu / \mathrm{kg} / \mathrm{h}$ and Propofol with doses of 20-30 mg bolus, and then 10-20 $\mu / \mathrm{kg} / \mathrm{min}$ infusion, with a control and preservation of BIS between 65 to 85, and RAMSY Sedation Score equal to 3, used. Loci of pins and head skin were anesthetized with idocaine. The total dose of local anaesthetic drugs was recorded at the end of the procedure. The time interval between drug discontinuation and start of tremor, as well as the time of emergence, is recorded as the time between relaxation and when the patient is able to cooperate for a neurological examination. All of the events during the operation and complications and complains of pain and restlessness were recorded.

After the completion of the procedure, the patients were transferred to (PACU). Post-operative pain and vital signs, hemodynamic changes, and respiratory and alert status of patients were investigated for 2 hours in PACU. Patients' pain was measured by using (VAS) (0: painless, 10: maximum pain) at intervals of 30 minutes, and in the case of VAS more than 3, $2 \mathrm{mg}$ morphine was injected intravenous in bolus and slowly. In analyzing data, the mean, standard deviations, frequency, tables and charts were used to categorize and summarize the collected data. In the study of statistical pre-requisites, considering the number of observations in each distribution, the Kolmogorov- 
Smirnov test was used to verify the natural distribution of the data. Regarding the existence of statistical hypotheses, independent t-test and Mann-Whitney test were used at 95\% confidence level and the statistical packet version 22 was used. In all analyzes, the significance level was considered as $\mathrm{p}<0.05$.

\section{Results}

The demographic characteristics of the participants in the study are presented in Table 1. Independent T-test showed that the two groups had no significant difference in terms of underlying variables (age and weight) and were similar. The mean age of patients was $53.08 \pm 10.29$ years and mean weight was $71.75 \pm 6.79 \mathrm{~kg}$.

Table 1. The characteristics of participants in the research

\begin{tabular}{cccccc}
\hline & Drug & Number & Mean & SD & P-VALUE \\
\hline \multirow{4}{*}{ Age } & DEX & 14 & 52.46 & 7.479 & $\mathbf{0 . 7 5 6}$ \\
& Propofol & 14 & 53.82 & 13.250 & \\
& Total & 28 & 53.08 & 10.29 & \\
& DEX & 14 & 72.38 & 5.679 & $\mathbf{0 . 3 8 5}$ \\
& Propofol & 14 & 69.91 & 7.981 & \\
& Total & 28 & 71.25 & 6.79 & \\
\hline
\end{tabular}

The results of Kolmogorov-Smirnov test showed that for three variables of BIS and time to cooperation and tremor, the distribution of variables was normal $(p>0.05)$ and for variables of VAS and surgeon satisfaction were not normal $(\mathrm{p}<0.05)$. Independent $\mathrm{t}$-test results showed that there was significant difference between Time to tremor and time to cooperation variables in the two groups (Table 2). In the group treated with Propofol, the mean time to tremor (11.36) was 6 times greater than that of the DEX group (5.08) (Fig. 1 and Table 2). Also, the average time to cooperation in the group treated with Propofol was greater than that of the DEX group (Table 2 and Figure 2). There was no significant difference between BIS in the two groups (Table 2 and Figure 3).

Table 2. The results of independent t-test in two groups and the results of variables comparison

\begin{tabular}{llcccc}
\hline & Drug & Number & Mean & SD & P-VALUE \\
\hline \multirow{2}{*}{ Time to tremor } & DEX & 14 & 5.08 & 1.320 & $\mathbf{0 . 0 0 1 *}^{*}$ \\
& Propofol & 14 & 11.36 & 1.567 & \\
& Total & 28 & 7.96 & 3.49 & \\
& Time to cooperation & 14 & 7.77 & 1.536 & $\mathbf{0 . 0 0 1}^{*}$. \\
& DEX & 14 & 17.09 & 3.590 & \\
& Propofol & 28 & 12.04 & 5.41 & \\
& Total & 14 & 72.08 & 1.891 & $\mathbf{0 . 0 7 4}$ \\
& DEX & 14 & 70.64 & 1.859 & \\
\hline
\end{tabular}

${ }^{* *} \mathrm{p}<0.001 * \mathrm{p}<0.05$

Also, the results of the Mann-Whitney test showed that there was a significant difference between VAS and surgeon satisfaction variables in the two groups (Table 3). The mean of VAS in the Propofol group was significantly higher than the DEX group (Table 3 and Figure 4). Mean surgeon satisfaction in the DEX group was significantly higher than Propofol group (Table 3 and Figure 5).

Table 3. The results of Man-Whitney test in two groups

\begin{tabular}{|c|c|c|c|c|}
\hline \multicolumn{5}{|c|}{ Ranks } \\
\hline & med1 & $\mathbf{N}$ & Mean Rank & P-VALUE \\
\hline \multirow[t]{2}{*}{ VAS } & DEX & 14 & 9.46 & $0.022 *$ \\
\hline & Propofol & 14 & 16.09 & \\
\hline \multirow[t]{2}{*}{ surgeon satisfaction } & DEX & 14 & 17.50 & $* * 0.001$ \\
\hline & Propofol & 14 & 6.59 & \\
\hline
\end{tabular}

** $\mathrm{p}<0.001 * \mathrm{p}<0.05$ 


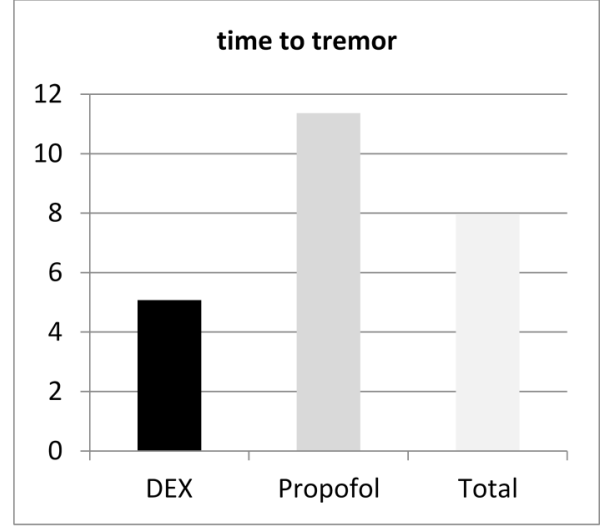

Figure 1. Time to tremor changes in two groups

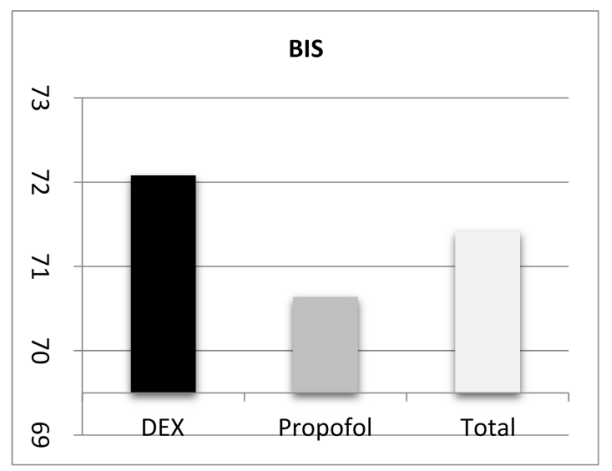

Figure 3. The changes of BIS in two groups surgeon satisfaction

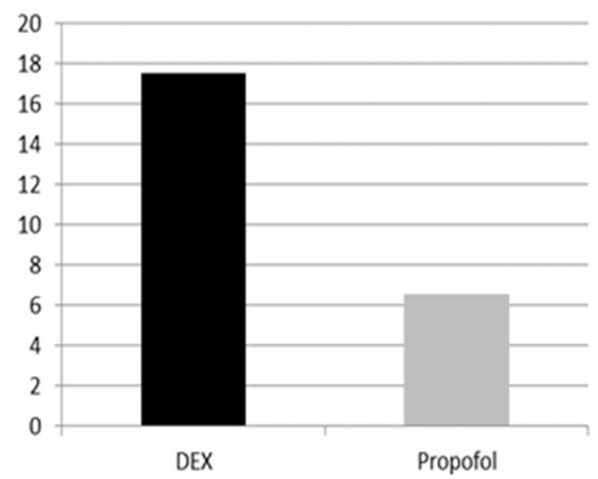

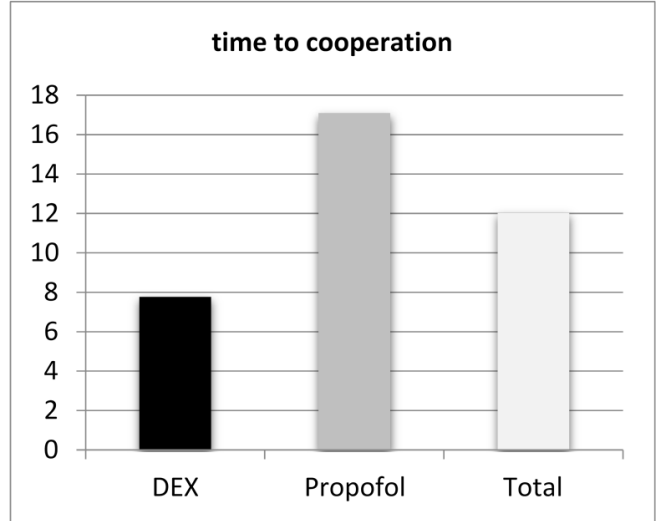

Figure 2. Time to cooperation changes in two groups

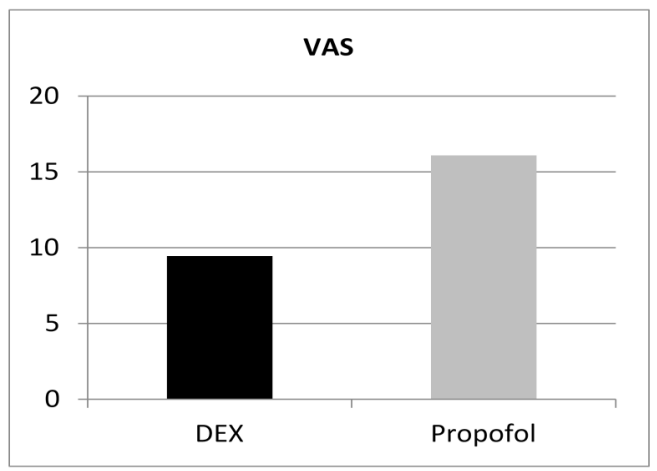

Figure 4. The changes of VAS in two groups

Figure 5. The changes of Surgeon satisfaction in two groups

\section{Discussion}

The purpose of this study was to investigate the effect of Dexmedetomidine and Propofol on the duration of onset of tremor after drug discontinuation in patients with Parkinson's disease under deep brain stimulation. Findings showed that there was a significant difference between time to tremor and time to cooperation variables in the two groups. The Mean comparison showed that the mean time to tremor and time to cooperation in the Propofol group was higher than the DEX group. Other findings showed that there was no significant difference between BIS in the two groups. The results also showed that there was a significant difference between VAS and surgeon satisfaction variables in the two groups. Mean VAS in Propofol group was significantly higher than DEX group. Indeed, mean surgeon satisfaction in the DEX group was significantly higher than Propofol group. 
According to the findings of the study, it can be mentioned that Dexmedetomidine has a better performance than Propofol. Some studies have documented Dexmedetomidine as an ideal anaesthetic drug in patients with deep brain stimulation, as it is easy for patients to be awakened and clinically evaluated during surgery (Schulz et al. al., 2007).

The findings of this study are not consistent with past researches. For example, Karabayırlı et al. (2015) examined the effects of Dexmedetomidine and Propofol on oxidative stress parameters during lower limb surgery. Their findings showed that there was no significant difference between the parameters of oxidative stress between the two groups. They concluded that Dexmedetomidine and Propofol had the same effects on the oxidative stress parameters during lower limb surgery (Karabayırlı, Uras, Koca, Ergin, \& Demircioglu, 2015). In another study, similar effects were reported with Dexmedetomidine and Propofol (Ünsal et al., 2004). Findings of the study was in line with the results of (Banihashem, SalehiOmran, \& Jalalian, 2015) (Shen et al., 2013), (Wang, Shi, Qin, Zhou, \& Xia, 2017).

Bani Hashem et al. (2015) examined the effect of sedation of Dexmedetomidine with midazolam in patients under esophageal echocardiography. Their findings showed that the level of analgesia was higher in patients with Dexmedetomidine than in the midazolam group. They concluded that Dexmedetomidine is a better drug than midazolam. Also, shen et al (2013) stated that Dexmedetomidine is better than Propofol, because in their study, patients had a shorter duration of arousal and more satisfaction than surgery. In another study, Wang et al (2017) compared the effects of Dexmedetomidine and Propofol on conscious sedation while repairing the hernia. Their findings showed that after prescribing sedative drugs, heart rate decreased significantly in patients receiving Dexmedetomidine. Compared to those receiving Propofol, the need for fentanyl surgery was significantly lower in patients receiving Dexmedetomidine. Administration of Dexmedetomidine was associated with a reduction in postoperative pain, a longer time to start relaxation and a lower recovery time. The patient's satisfaction with Dexmedetomidine can be considered as the degree of tranquility of patients in this group compared to the Propofol group. Patients in the Dexmedetomidine group experienced less pain and this resulted in more satisfaction. Dexmedetomidine is a specific alpha-2 receptor agonist that has been used successfully as an analgesic and analgesic agent in surgery such as craniotomy, fiber optic bronchoscopy, and other diagnostic tests through the recent years. Although, bradycardia and hypotension have been reported as adverse effects of Dexmedetomidine administration in recent studies (Carollo, Nossaman, \& Ramadhyani, 2008), the present study did not show any adverse events in hemodynamic events due to the relatively low dose of Dexmedetomidine. According to studies published on the beneficial effects of Dexmedetomidine, its role in anaesthetic is increasing day by day. As studies have shown that the incidence of nausea, vomiting, irritation and tremor has decreased in the postoperative period (Cheung et al., 2007; Isik, Arslan, Tunga, \& Kurtipek, 2006; Yildiz et al., 2006). Dexmedetomidine is an effective and sedative drug. All of these factors make Dexmedetomidine as an attractive alternative to sedation, when the patient needs self-contained breathing and comfort from relaxation.

\section{Conclusion}

Regarding the obtained results, it can be said that for deep brain excitation in patients with Parkinson's, Dexmedetomidine is the superior drug to Propofol. Therefore, it can be suggested to anesthesiologists to use this drug to treat Parkinson's patients.

\section{References}

Anderson, B., Marks, P., \& Futter, M. (1994). Propofol-contrasting effects in movement disorders. British journal of neurosurgery, 8(3), 387-388 .

Banihashem, N., SalehiOmran, M., \& Jalalian, R. (2015). Sedative Effect of Dexmedetomidine and Midazolam in Transesophageal Echocardiographic Examination. Journal of Mazandaran University of Medical Sciences, 24(122), 130-136 .

Böhmdorfer, W., Schwarzinger, P., Binder, S., \& Sporn, P. (2003). Temporary suppression of tremor by remifentanil in a patient with Parkinson's disease during cataract extraction under local anesthesia. Der Anaesthesist, 52(9), 795-797.

Carollo, D. S., Nossaman, B. D., \& Ramadhyani, U. (2008). Dexmedetomidine: a review of clinical applications. Current Opinion in Anesthesiology, 21(4), 457-461.

Cheung, C. W., Ying, C. L. A., Chiu, W. K., Wong, G. T., Ng, K. F. J., \& Irwin, M. G. (2007). A comparison of dexmedetomidine and midazolam for sedation in third molar surgery. Anaesthesia, 62(11), 1132-1138 .

Fábregas, N., Rapado, J., Gambús, P .L., Valero, R., Carrero, E., Salvador, L., . . Trocóniz, I. F. (2002). Modeling of the sedative and airway obstruction effects of propofol in patients with Parkinson disease undergoing stereotactic surgery. The Journal of the American Society of Anesthesiologists, 97(6), 1378-1386 . 
Finelli, D. A., Rezai, A. R., Ruggieri, P. M., Tkach, J. A., Nyenhuis, J. A., Hrdlicka, G., . . Shellock, F. G. (2002). MR imaging-related heating of deep brain stimulation electrodes: in vitro study. American Journal of Neuroradiology, 23(10), 1795-1802 .

Guldenmund, P., Vanhaudenhuyse, A., Sanders, R., Sleigh, J., Bruno, M., Demertzi, A., . . Baquero, K. (2017). Brain functional connectivity differentiates dexmedetomidine from propofol and natural sleep. BJA: British Journal of Anaesthesia, 119(4), 674-684 .

Isik, B., Arslan, M., Tunga, A. D., \& Kurtipek, O. (2006). Dexmedetomidine decreases emergence agitation in pediatric patients after sevoflurane anesthesia without surgery. Pediatric Anesthesia, 16(7), 748-753 .

Karabayırl, S., Uras, I., Koca, C., Ergin, M., \& Demircioglu, R. (2015). The Effect of Dexmedetomidine and Propofol on Oxidative Stress Parameters during Lower Extremity Surgery: A Prospective Randomized Trial. Int $J$ anaesthetic Anesthesiol, 2, 034 .

Mack ,P. F., Perrine, K., Kobylarz, E., Schwartz, T. H., \& Lien, C. A. (2004). Dexmedetomidine and neurocognitive testing in awake craniotomy. Journal of neurosurgical anesthesiology, 16(1), 20-25 .

Maltête, D., Navarro, S., Welter, M.-L., Roche, S., Bonnet, A.-M., Houeto, J.-L., . . Cornu, P. (2004). Subthalamic stimulation in Parkinson disease: with or without anesthesia? Archives of neurology, 61(3), 390-392 .

Martinez-Simon, A., Alegre, M., Honorato-Cia, C., Nuñez-Cordoba, J. M., Cacho-Asenjo, E., Trocóniz, I. F., .. . Guridi, J. (2017). Effect of Dexmedetomidine and Propofol on Basal Ganglia Activity in Parkinson DiseaseA Controlled Clinical Trial. Anesthesiology: The Journal of the American Society of Anesthesiologists, 126(6), 1033-1042.

Rozet, I. (2008). Anesthesia for functional neurosurgery: the role of dexmedetomidine. Current Opinion in Anesthesiology, 21(5), 537-543 .

Rozet, I., Muangman, S., Vavilala, M. S., Lee, L. A., Souter, M. J., Domino, K. J., . . Lam, A. M. (2006). Clinical experience with dexmedetomidine for implantation of deep brain stimulators in Parkinson's disease. Anesthesia \& Analgesia, 103(5), 1224-1228.

Schilling, J. M., Singaram, V., Dhanani, M., Habib, A. M., Bui, J. D., Patel, H. H., \& Meier, A. (2017). Dexmedetomidine and propofol differentially alter human immune cell metabolism. The FASEB Journal, 31(1 Supplement), 1000.1004-1000.1004 .

Schulz, U., Keh, D., Barner, C., Kaisers, U., \& Boemke, W. (2007). Bispectral index monitoring does not improve anesthesia performance in patients with movement disorders undergoing deep brain stimulating electrode implantation. Anesthesia \& Analgesia, 104(6), 1481-1487.

Shen, S.-1., Zheng, J.-y., Zhang, J., Wang, W.-y., Jin, T., Zhu, J., \& Zhang, Q. (2013). Comparison of dexmedetomidine and propofol for conscious sedation in awake craniotomy: a prospective, double-blind, randomized, and controlled clinical trial. Annals of Pharmacotherapy, 47(11), 1391-1399 .

Souter, M. J., Rozet, I., Ojemann, J. G., Souter, K. J., Holmes, M. D., Lee, L., \& Lam, A. M. (2007). Dexmedetomidine sedation during awake craniotomy for seizure resection: effects on electrocorticography. Journal of neurosurgical anesthesiology, 19(1), 38-44 .

Ünsal, A., Devrim, E., Guven, C., Eroglu, M., Durak, I., Bozoklu ,A., \& Balbay, M. D. (2004). Propofol attenuates reperfusion injury after testicular torsion and detorsion. World journal of urology, 22(6), 461-465 .

Wang, H.-M., Shi, X.-Y., Qin, X.-R., Zhou, J.-L., \& Xia, Y.-F. (2017). Comparison of dexmedetomidine and propofol for conscious sedation in inguinal hernia repair: A prospective, randomized, controlled trial. Journal of International Medical Research, 45(2), 533-539.

Yang, G.-Z., Xue, F.-S., \& Sun, C. (2017). Assessing interaction between dexmedetomidine and propofol. Journal of anesthesia, 31(1), 156-156 .

Yildiz, M., Tavlan, A., Tuncer, S., Reisli, R., Yosunkaya, A., \& Otelcioglu, S. (2006). Effect of dexmedetomidine on haemodynamic responses to laryngoscopy and intubation. Drugs in $R \& D, 7(1), 43-52$.

\section{Copyrights}

Copyright for this article is retained by the author(s), with first publication rights granted to the journal.

This is an open-access article distributed under the terms and conditions of the Creative Commons Attribution license (http://creativecommons.org/licenses/by/4.0/). 